\title{
Harmonic enhancement of single-bubble sonoluminescence
}

\author{
Xiaozhen Lu, ${ }^{1}$ Andrea Prosperetti, ${ }^{1,2, *}$ Ruediger Toegel, ${ }^{2}$ and Detlef Lohse ${ }^{2}$ \\ ${ }^{1}$ Department of Mechanical Engineering, The Johns Hopkins University, Baltimore, Maryland 21218 \\ ${ }^{2}$ Department of Applied Physics and J.M. Burgers Centre for Fluid Dynamics, University of Twente, \\ AE 7500 Enschede, The Netherlands
}

(Received 12 December 2002; published 22 May 2003)

\begin{abstract}
It is known from experiment that the light emission from a sonoluminescing bubble can be increased by using more than one driving frequency. In this paper, a systematic method to determine the optimal conditions of pressure amplitude and relative phase for this effect is described. As a specific application, a two-frequency system-26.5 kHz and $53 \mathrm{kHz}$-is considered. It is found that the maximum temperatures achievable can be appreciably increased with respect to single-frequency drive, still maintaining spherical stability, provided the dissolved inert gas concentration is kept extremely low in order to maintain diffusive stability.
\end{abstract}

DOI: 10.1103/PhysRevE.67.056310

PACS number(s): 78.60.Mq, 47.20.Ma, 47.55.Dz

\section{INTRODUCTION}

The remarkable phenomenon of single-bubble sonoluminescence $[1,2]$ consists of the periodic light emission from a gas bubble driven into radial pulsation by a sound field (for a recent review, see Ref. [3]). The numerous puzzling features reported by the early investigators of the phenomenon (see, e.g., Ref. [4]) have found a satisfactory explanation in subsequent work [3], which agrees very well with experiment. Briefly, the light is due to a weakly ionized plasma that forms in the bubble due to the intense, nearly adiabatic compression of the gas that takes place during the bubble collapse [3].

In view of the surprising intensity of the phenomenon, it is of considerable interest to try to further enhance sonoluminescence emission by increasing the sound field amplitude. Unfortunately, this objective is difficult to achieve since, at high driving amplitudes, the spherical shape becomes unstable, which leads to the fragmentation and ultimate destruction of the bubble [5-8]. Another approach that has been followed to achieve the same objective has been the use of a lower-frequency drive which, however, has proven equally unsuccessful due to the accumulation of water vapor inside the bubble [9-12].

While most of the work to date has been carried out with the bubble driven by a monochromatic sound field, some investigators have been experimenting with multifrequency acoustic drives [13-18]. Of particular interest is the observation of Refs. $[13,15]$ which report an increase by up to $300 \%$ of the emitted light intensity with a dual-frequency drive. Since this result was reached by varying the relative phase of the two harmonics of the sound field by trial and error, it is natural to enquire whether, by a systematic investigation of the matter, it would be possible to further increase the light emission. This idea was investigated in a conference paper [19], of which the present work is an extension and an elaboration. Our conclusion is that a further enhancement appears indeed possible. Specifically, we consider a sound field consisting of two harmonics and maximize the bubble's

\footnotetext{
*Corresponding author.
}

peak temperature, under the condition that the spherical stability of the bubble be preserved. Although here we limit ourselves to two frequencies, the method we describe is general and can be adapted to a greater number of monochromatic components.

\section{MATHEMATICAL MODEL}

The model we use for the bubble dynamics and thermodynamics is basically that of Refs. [20,21], which has proven to accurately account for various experimental phase diagrams $[3,21]$. It is very similar to the model by Storey and Szeri $[10,22]$. We consider an argon bubble in water including the effects of water vapor diffusion, conductive heat loss, and chemical reactions.

\section{A. Bubble dynamics}

The radial motion of the bubble is described by a variant of the Rayleigh-Plesset equation taking into account firstorder corrections for the liquid compressibility [23].

$$
\begin{aligned}
\left(1-\frac{\dot{R}}{c}\right) R \ddot{R}+\frac{3}{2} \dot{R}^{2}\left(1-\frac{\dot{R}}{3 c}\right)= & \left(1+\frac{\dot{R}}{c}\right) \frac{1}{\rho}\left(p_{g}-P_{a}-P_{0}\right) \\
& +\frac{R}{\rho c} \dot{p}_{g}-4 \nu \frac{\dot{R}}{R}-\frac{2 \sigma}{\rho R} .
\end{aligned}
$$

Here, dots denote time differentiation, $\rho, c, \sigma$, and $\mu$ are the density, speed of sound, surface tension coefficient, and viscosity of the liquid, $p_{g}$ is the bubble internal pressure, $P_{0}$ is the static pressure, and $P_{a}(t)$ is the acoustic driving pressure, for which we assume the form

$$
P_{A}=p_{1} \cos \omega_{1} t+\sum_{\ell=2}^{N}\left(p_{\ell} \cos \omega_{\ell} t+q_{\ell} \sin \omega_{\ell} t\right)
$$

with the time origin chosen in such a way that $q_{1}=0$. The frequencies $\omega_{k}$ depend on the apparatus and are considered prescribed.

The internal pressure $p_{g}$ is modeled by a van der Waals type equation of state, 


$$
p_{g}=\frac{N_{\text {tot }} k T}{V-N_{\text {tot }} B}
$$

with $N_{\text {tot }}$ the total number of particles (i.e., argon atoms, vapor and its reaction products) and $B=5.1 \times 10^{29} \mathrm{~m}^{3}$ [24] the covolume, which-for simplicity-is taken to be equal for all species.

\section{B. Mass transport}

The number of particles of species $i$ in the bubble change with time because of diffusion and chemical reactions. We model the diffusive rate of change $\dot{N}_{i}^{d}$ by means of the boundary layer approach of Ref. [12].

$$
\dot{N}_{i}^{d}=4 \pi R^{2} D \frac{n_{i, 0}-n_{i}}{l_{d}}, \quad l_{d}=\min \left(\sqrt{\frac{R D}{|\dot{R}|}}, \frac{R}{\pi}\right) .
$$

Here, $n_{i}$ and $n_{i, 0}$ are the instantaneous and equilibrium concentration of particles of species $i$, respectively; $D$ is the binary diffusion coefficient of the water vapor-argon mixture and $l_{d}$ is the thickness of the diffusive boundary layer. The previous approximation to $l_{d}$ is only valid in the regime $P e_{D}=R|\dot{R}| / D>1$. Therefore, $l_{d}$ is not allowed to become smaller then $R / \pi$ (see Ref. [12] for details). Note that we use a common diffusion constant and correspondingly a common $l_{d}$ for all species. This simplification has proven sufficient in our earlier work.

Because of the large heat capacity of water we will assume isothermal behavior at the bubble wall. The diffusion constant $D$ is correspondingly given by scaling its value under normal conditions $(101.3 \mathrm{kPa}, 293.15 \mathrm{~K})$ with the number density in the boundary layer, the composition of which is assumed to be dominated by argon and vapor in equilibrium with the liquid phase. Hence, $D=D_{0}\left[n_{0} /\left(n_{\mathrm{H}_{2} \mathrm{O}, 0}\right.\right.$ $\left.\left.+n_{\mathrm{Ar}}\right)\right]$, where $D_{0}=23.55 \times 10^{-6} \mathrm{~m}^{2} / \mathrm{s}$ [25] and $n_{0}=2.446$ $\times 10^{25} \mathrm{~m}^{-3}$. Finally, in order to completely specify Eq. (4), the equilibrium concentrations $n_{i, 0}$ at the bubble wall must be set. For $\mathrm{H}_{2} \mathrm{O}$ it is given by the number density corresponding to the saturation vapor pressure at temperature $T_{0}: n_{\mathrm{H}_{2} \mathrm{O}, 0}$ $=P_{\mathrm{v}}\left(T_{0}\right) / k T_{0} \approx 5.9 \times 10^{23} \mathrm{~m}^{-3}$. For all other species we simply set $n_{i, 0}=0$, as in the situation of harmonic driving the liquid must be highly undersaturated in order to achieve diffusive stability of the bubble, cf. Table IV.

\section{Heat loss}

Analogously to Eq. (4), we approximate the conductive heat loss by

$$
\dot{Q}=4 \pi R^{2} \kappa \frac{T_{0}-T}{l_{\mathrm{th}}}, \quad l_{\mathrm{th}}=\min \left(\sqrt{\frac{R \chi}{|\dot{R}|}}, \frac{R}{\pi}\right),
$$

$T$ being the temperature of the bubble contents, $T_{0}$ the (liquid) temperature at the bubble wall, $\kappa$ the thermal conductivity of the gas mixture, $\chi$ its thermal diffusivity, and $l_{\mathrm{th}}$ the thickness of the thermal boundary layer. An effective heat conductivity is obtained from the empirical formula [26]

$$
\begin{gathered}
\kappa_{\operatorname{mix}}=\sum_{i} \frac{\xi_{i} \kappa_{i}}{\sum_{j} \xi_{j} \Phi_{i, j}}, \\
\Phi_{i, j}=\frac{1}{\sqrt{8}}\left(1+\frac{m_{i}}{m_{j}}\right)^{-1 / 2}\left[1+\left(\frac{\eta_{i}}{\eta_{j}}\right)^{1 / 2}\left(\frac{m_{j}}{m_{i}}\right)^{1 / 4}\right]^{2},
\end{gathered}
$$

which relates the heat conductivities $\lambda_{i}$ and viscosities $\eta_{i}$ of the pure substances to the conductivity of the mixture. $\xi_{i}$ denotes the mole fraction of species $\mathrm{i}$ and $m_{i}$ its molecular mass. In our case $\kappa_{\mathrm{Ar}}=17.8 \times 10^{-3} \mathrm{~W} / \mathrm{mK}, \kappa_{\mathrm{H}_{2} \mathrm{O}}=18$ $\times 10^{-3} \mathrm{~W} / \mathrm{mK}, \quad \eta_{\mathrm{Ar}}=22.8 \times 10^{-6} \mathrm{Pas}, \quad$ and $\eta_{\mathrm{H}_{2} \mathrm{O}}=10$ $\times 10^{-6} \mathrm{Pas}$ [24]. The thermal diffusivity is finally obtained from $\chi=\kappa_{\text {mix }} / c_{p}$, with $c_{p}=\frac{5}{2} n_{\mathrm{Ar}} k+\frac{8}{2} n_{\mathrm{H}_{2} \mathrm{O}, 0} k$ the constantpressure heat capacity per unit volume of the gas mixture at the wall.

\section{Chemical reactions}

The rates of the chemical reactions are described by means of modified Arrhenius laws. Following Ref. [20], we furthermore include a correction factor in the forward reaction rate of every elementary reaction to approximately account for the shift of the equilibrium constant under high density. The general form for the reaction rates of a chemical process $M+A+B \leftrightarrow M+C+D$ then becomes

$$
\begin{gathered}
r_{f, j}=\left[\frac{\exp \left[n_{\mathrm{tot}} B /\left(1-n_{\mathrm{tot}} B\right)\right]}{1-n_{\mathrm{tot}} B}\right]^{t_{\mathrm{j}}} k_{f, j} n_{\mathrm{tot}} n_{A} n_{B} T^{c_{f, j}} \\
\times \exp \left(-\frac{E_{f, j}}{k T}\right), \\
r_{b, j}=k_{b, j} n_{\mathrm{tot}} n_{C} n_{D} T^{c_{b, j}} \exp \left(-\frac{E_{b, j}}{k T}\right), \\
r_{j}=r_{f, j}-r_{b, j},
\end{gathered}
$$

with $n_{A, \ldots, D}$ the concentration of the participating species, $n_{\text {tot }}$ the number density of the collider $M$ (given by the total number density as every particle can act as a collider), and $r_{j}$ the net reaction rate per unit volume given by the difference between the forward and backward rates $r_{f, j}$ and $r_{b, j}$. Note that for reactions of type $A+B \leftrightarrow C+D$ or $M+A+B \leftrightarrow M$ $+C$ the concentration must be adapted accordingly. Table I lists the parameters used [45]. The chemical rate of change of species $i$ is now given by the sum over all elementary reaction rates with their corresponding stoichiometric weight $\alpha_{i, j}$

$$
\dot{N}_{i}^{c}=V \sum_{j} \alpha_{i, j} r_{i}
$$

To illustrate this consider, for example, reaction $j=1$. In this reaction oxygen radicals $(i=\mathrm{O})$ have a stoichiometric weight $\alpha_{\mathrm{O}, 1}=-2$, since two $\mathrm{O}$ radicals are destroyed in the process. For say hydroxyl radicals $(i=\mathrm{OH})$ it is obviously $\alpha_{\mathrm{OH}, 1}=0$, since hydroxyl radicals are not involved in reaction $j=1$. 
TABLE I. Arrhenius parameters of the reaction scheme $[24,45]$. The frequency factors $k_{f, j}, k_{b, j}$ are given in $\mathrm{cm}^{3}(\mathrm{~mol} / \mathrm{s})$ for the two-body reactions and in $\mathrm{cm}^{6}\left(\mathrm{~mol}^{2} / \mathrm{s}\right)$ for the three-body reactions. $E_{f, j} / k$ and $E_{b, j} / k$ are given in $\mathrm{K}$ and the reaction energies $\Delta E_{\mathrm{j}}$ are in $\mathrm{kJ} / \mathrm{mol}$.

\begin{tabular}{cccccccccc}
\hline \hline No. & Reaction & $t_{j}$ & $k_{f, j}$ & $c_{f, j}$ & $E_{f, j} / k$ & $k_{b, j}$ & $c_{b, j}$ & $E_{b, j} / k$ & $\Delta E_{j}$ \\
\hline 1 & $\mathrm{O}+\mathrm{O}+\mathrm{M} \leftrightarrow \mathrm{O}_{2}+\mathrm{M}$ & 1 & $1.2 \times 10^{17}$ & -1 & 0 & $3.16 \times 10^{19}$ & -1.3 & 59893 & 498 \\
2 & $\mathrm{O}+\mathrm{H}+\mathrm{M} \leftrightarrow \mathrm{OH}+\mathrm{M}$ & 1 & $5 \times 10^{17}$ & -1 & 0 & $3.54 \times 10^{17}$ & -0.9 & 51217 & 428 \\
3 & $\mathrm{O}+\mathrm{H}_{2} \leftrightarrow \mathrm{H}+\mathrm{OH}$ & 0 & $3.87 \times 10^{4}$ & 2.7 & 3150 & $1.79 \times 10^{4}$ & 2.7 & 2200 & -8 \\
4 & $\mathrm{H}+\mathrm{O}_{2} \leftrightarrow \mathrm{O}+\mathrm{OH}$ & 0 & $2.65 \times 10^{16}$ & -0.7 & 8576 & $9 \times 10^{13}$ & -0.3 & -83 & -70 \\
5 & $\mathrm{H}+\mathrm{H}+\mathrm{M} \leftrightarrow \mathrm{H}_{2}+\mathrm{M}$ & 1 & $1 \times 10^{18}$ & -1 & 0 & $7.46 \times 10^{17}$ & -0.8 & 52177 & 436 \\
6 & $\mathrm{H}+\mathrm{OH}+\mathrm{M} \leftrightarrow \mathrm{H}_{2} \mathrm{O}+\mathrm{M}$ & 1 & $2.2 \times 10^{22}$ & -2 & 0 & $3.67 \times 10^{23}$ & -2 & 59980 & 498 \\
7 & $\mathrm{OH}+\mathrm{H}_{2} \leftrightarrow \mathrm{H}+\mathrm{H}_{2} \mathrm{O}$ & 0 & $2.16 \times 10^{8}$ & 1.5 & 1726 & $5.2 \times 10^{9}$ & 1.3 & 9529 & 62 \\
8 & $\mathrm{OH}+\mathrm{OH} \leftrightarrow \mathrm{O}+\mathrm{H}_{2} \mathrm{O}$ & 0 & $3.57 \times 10^{4}$ & 2.4 & 1062 & $1.74 \times 10^{6}$ & 2.2 & 7693 & 70 \\
\hline \hline
\end{tabular}

Given the chemical and diffusive rate of change by Eq. (10) and Eq. (4), the total rate of change of species $i$ eventually becomes

$$
\dot{N}_{i}=\dot{N}_{i}^{d}+\dot{N}_{i}^{c}
$$

\section{E. Energy balance}

In order to derive a differential equation for the gas temperature, we start from the global energy balance of the bubble interior [27]

$$
\dot{E}=\dot{Q}-p_{\mathrm{g}} \dot{V}+\sum_{i}\left(h_{w, i}+h_{\text {form }, i}\right) \dot{N}_{i}^{d}
$$

Here, $\dot{E}$ is the rate of change of the total energy, $\dot{Q}$ is the conductive heat loss, $p_{\mathrm{g}} \dot{V}$ the work performed on the bubble, and $\sum_{i}\left(h_{w, i}+h_{\text {form }, i}\right) \dot{N}_{i}^{d}$ is the energy loss due to diffusion. The terms in the summation account for the enthalpy of formation $h_{\text {form, } i}$ of the various radicals; differences between these enthalpies determine the reaction energy of the chemical reactions. Hence, we write

$$
E=\sum_{i}\left(e_{\mathrm{th}, i}+h_{\mathrm{form}, i}\right) N_{i}
$$

with the thermal energy per molecule $e_{\text {th,i }}$ given by

$$
e_{\mathrm{th}, i}=\frac{f_{i}}{2} k T+\sum_{l} \frac{k \Theta_{i, l}}{\Theta_{i, l}}-1 \text {. }
$$

$f_{i}$ is the number of translational and rotational degrees of freedom and $\Theta_{i, l}$ the characteristic vibrational temperature of species $i$. Table II lists the values used in the calculation [46]. Taking the time derivative of Eq. (13) one finds

$$
\begin{aligned}
\dot{E}= & \dot{T} \sum_{i} \frac{\partial e_{\mathrm{th}, i}}{\partial T} N_{i}+\sum_{i} h_{\mathrm{form}, i} \dot{N}_{i}^{c}+\sum_{i} e_{\mathrm{th}, i} \dot{N}_{i}^{c}+\sum_{i} h_{\mathrm{form}, i} \dot{N}_{i}^{d} \\
& +\sum_{i} e_{\mathrm{th}, i} \dot{N}_{i}^{d} .
\end{aligned}
$$

The second term in the last equation is readily recognized as the net reaction energy per unit time:

$$
\sum_{i} h_{\text {form }, i} \dot{N}_{i}^{c}=-V \sum_{j} r_{j} \Delta E_{j} .
$$

Upon equating Eq. (15) and Eq. (12) and using this relation, we finally obtain a differential equation for the temperature $T$. It will be noted that, as expected, the enthalpies of formation $h_{\text {form, } i}$ are thus only important in the net reaction energy, but not in the final temperature equation.

We note that our model does not include ionization reactions or electronic excitation even though, for the smaller bubbles that we simulate, we find peak temperatures of the order of ten times the ionization temperature. Inclusion of these effects would considerably lower the peak temperatures as presumably a major part of the compressional work would be consumed by them. Although our predictions may not be quite realistic in these cases, it should be noted that the problem does not even arise in the case of singlefrequency driving as the calculated temperatures are much lower. The trend toward a much increased temperature is therefore a robust prediction.

\section{F. Diffusional stability}

In order to prevent the disappearance of the bubble, the maximization of the temperature must be effected under the constraints that the bubble maintain (1) diffusional and (2) shape stability. The former condition translates to

TABLE II. Number of translational+rotational degrees of freedom and characteristic vibrational temperatures of the various species $[24,46]$. Note also that $h_{w, i}=\left(f_{i}+2\right) / 2 k T_{0}$.

\begin{tabular}{cccccccc}
\hline \hline Species & $\mathrm{H}_{2}$ & $\mathrm{H}$ & $\mathrm{O}$ & $\mathrm{O}_{2}$ & $\mathrm{OH}$ & $\mathrm{H}_{2} \mathrm{O}$ & $\mathrm{Ar}$ \\
\hline$\Theta_{i, l}$ & 6325 & & & 2273 & 5370 & 2295 & \\
$\Theta_{i, 2}$ & & & & & & 5255 & \\
$\Theta_{i, 3}$ & & & & & & 5400 & \\
$\mathrm{f}_{i}$ & 5 & 3 & 3 & 5 & 5 & 6 & 3 \\
\hline \hline
\end{tabular}


TABLE III. Compression ratio $s:=R_{\mathrm{Max}} / R_{\min }$, pressure amplitudes $p_{1}, p_{2}, q_{2}$, normalized equilibrium dissolved gas concentration $C_{\infty} / C_{\text {sat }}$, and estimated maximum gas temperature $T_{\text {Max }}$, for single-frequency drive (left) and optimal two-frequency drive for the cases shown in Fig. 1; $C_{\text {sat }}$ is the saturation concentration. The calculations are for an argon-water system at standard temperature and pressure.

\begin{tabular}{ccccccccccc}
\hline \hline \multicolumn{1}{c}{ Single-frequency drive } & \multicolumn{7}{c}{ Multifrequency drive } \\
$R_{0}$ & $s$ & $\begin{array}{c}p_{1} \\
(\mu \mathrm{m})\end{array}$ & $C_{\infty} / C_{\text {sat }}$ & $T_{\text {Max }}$ & $s$ & $p_{1}$ & $p_{2}$ & $q_{2}$ & $C_{\infty} / C_{\text {sat }}$ & $T_{\text {Max }}$ \\
\hline 2.0 & 545 & 316 & $4.3 \times 10^{-6}$ & 25100 & 955 & 48 & -775 & -1605 & $1.14 \times 10^{-6}$ & 688000 \\
2.5 & 404 & 289 & $9.9 \times 10^{-6}$ & 23500 & 738 & 32 & -1597 & -519 & $2.22 \times 10^{-6}$ & 451000 \\
3.0 & 267 & 243 & $2.86 \times 10^{-5}$ & 21500 & 633 & 16.3 & 1306 & 108 & $3.7 \times 10^{-6}$ & 319000 \\
3.5 & 145 & 196 & $1.08 \times 10^{-4}$ & 14600 & 446 & 120 & -954 & 84 & $9.75 \times 10^{-6}$ & 153000 \\
4.0 & 116 & 171 & $3.2 \times 10^{-4}$ & 11300 & 220 & 240 & -76 & -75 & $5.15 \times 10^{-5}$ & 17400 \\
4.5 & 88 & 153 & $9.00 \times 10^{-4}$ & 10400 & 177 & 222 & -71 & -100 & $8.7 \times 10^{-5}$ & 15400 \\
5.0 & 62 & 138 & $2.56 \times 10^{-3}$ & 9600 & 224 & 288 & -87 & -9 & $5.0 \times 10^{-5}$ & 14700 \\
\hline \hline
\end{tabular}

$$
\left.\frac{\partial C_{\infty}}{\partial R_{0}}\right|_{\dot{m}=0}>0
$$

where $C_{\infty}$ is the gas concentration in the liquid; the derivative is taken along the line of diffusional equilibrium along which $\dot{m}$, the net gas inflow into the bubble over one cycle, vanishes. The condition (17) ensures that, for a given $C_{\infty}$, a small increase in the equilibrium radius (which is related to the mass of gas inside the bubble) will bring the bubble into a region where gas diffuses out of it thus restoring the original equilibrium radius, and conversely for a small $R_{0}$ decrease. The dissolved concentration $C_{\infty}$ with which a bubble of equilibrium radius $R_{0}$ is in diffusional equilibrium is given by [28]

$$
\frac{C_{\infty}}{C_{\mathrm{sat}}}=\frac{1}{P_{\infty}} \frac{\left\langle p(t) R^{4}\right\rangle}{\left\langle R^{4}\right\rangle},
$$

where $C_{\text {sat }}$ is the saturation concentration at $P_{\infty}$ and the angular brackets indicate the average over a complete period of the sound pressure amplitude (2).

\section{G. Shape stability}

In order to check the shape stability of the bubble, we have recourse to the equation governing the initial growth of a shape distortion proportional to the $n$th order spherical harmonic, which is (see, e.g., Refs. [6,7,29,30])

$$
\begin{aligned}
\ddot{a}_{n}+ & {\left[3 \frac{\dot{R}}{R}-2(n-1)(n+1)(n+2) \frac{\mu}{\rho R^{2}}+2 \frac{n(n+2)^{2}}{1+2 \delta / R} \frac{\mu}{\rho R^{2}}\right] } \\
& \times \dot{a}_{n}+(n-1)\left[-\frac{\ddot{R}}{R}+(n+1)(n+2) \frac{\sigma}{\rho R^{3}}\right. \\
& \left.+2(n+2) \frac{\mu \dot{R}}{\rho R^{3}}\left(n+1-\frac{n}{1+2 \delta / R}\right)\right] a_{n}=0
\end{aligned}
$$

Here, $a_{n}$ is the amplitude of the shape distortion and $\delta$ is the viscous boundary layer thickness approximated by $[6,31]$

$$
\delta=\min \left(\sqrt{\frac{\mu}{\rho \omega_{1}}}, \frac{R}{2 n}\right) .
$$

At steady state the coefficients of Eq. (19) are periodic functions of time and, therefore, Floquet theory applies (see, e.g., Ref. [32]), according to which the solution $\left(a_{n}, \dot{a}_{n}\right)$ at the end of a cycle is linearly related to $\left(a_{n}, \dot{a}_{n}\right)$ at the beginning of the cycle. It can be shown that, when the eigenvalues of the matrix establishing this linear relationship are complex, the spherical shape is stable. When they are real, let $\lambda$ be the one with the greater modulus; then, if $|\lambda|>1,\left(a_{n}, \dot{a}_{n}\right)$ will grow over each cycle and the bubble will be shape unstable $[5,6]$.

\section{NUMERICAL METHODS}

An exploratory calculation readily shows that, considered as a function of the pressure amplitudes $\left(p_{k}, q_{k}\right)$ for a given bubble equilibrium radius $R_{0}$, the maximum temperature $T_{\text {max }}$ possesses a great many points of relative maximum and minimum, cf. also Tables III and IV. This circumstance renders the more straightforward optimization algorithms, such as Newton-Raphson, ineffective. For this reason, we have chosen simulated annealing, which has the virtue of allowing

TABLE IV. Result of the optimization process, with an annealing rate three times as large as in Table III; all other parameters have the same values as before.

\begin{tabular}{ccccccc}
\hline \hline \multicolumn{7}{c}{ Multifrequency drive } \\
$R_{0}$ & $s$ & $p_{1}$ & $p_{2}$ & $q_{2}$ & $C_{\infty} / C_{\text {sat }}$ & $T_{\text {Max }}$ \\
$(\mu \mathrm{m})$ & & $(\mathrm{kPa})$ & $(\mathrm{kPa})$ & $(\mathrm{kPa})$ & & $(\mathrm{K})$ \\
\hline 2.0 & 2255 & 2831 & 14,580 & -6411 & $3.82 \times 10^{-8}$ & 1466000 \\
2.5 & 722 & 213 & -488 & -1546 & $1.85 \times 10^{-6}$ & 436000 \\
3.0 & 539 & 164 & -847 & -1049 & $3.9 \times 10^{-6}$ & 293000 \\
3.5 & 275 & 208 & -219 & 63 & $2.6 \times 10^{-5}$ & 21500 \\
4.0 & 242 & 217 & -185 & 50 & $3.8 \times 10^{-5}$ & 18300 \\
4.5 & 164 & -246 & 53 & -13 & $1.11 \times 10^{-4}$ & 14700 \\
5.0 & 96 & 99 & -107 & -119 & $4.6 \times 10^{-5}$ & 10900 \\
\hline \hline
\end{tabular}


the search process to escape local extrema. The implementation of the algorithm that we use is that described in Ref. [33], which can be summarized as follows.

Start with a set of values for the amplitudes $\left(p_{k}, q_{k}\right)$ in the shape-stable region and calculate the corresponding value of $u=\ln \left(T_{\max }\right)$ for steady state oscillations of the bubble. We optimize the logarithm of the temperature rather than the temperature itself, as the latter one spans orders of magnitude and therefore is not a suitable objective function. A random number generator produces a new set of amplitudes $\left(p_{k}^{\prime}, q_{k}^{\prime}\right)$ which is used to calculate a new $u^{\prime}$ at steady state. We first test whether the corresponding prolate-oblate distortion amplitude $a_{2}$ of the bubble is stable or unstable by calculating its largest Floquet multiplier from Eq. (19). In the latter case, the set is discarded (and counted as a failed step), a new set $\left(p_{k}^{\prime}, q_{k}^{\prime}\right)$ is generated, and shape stability is tested again. If, instead, the set $\left(p_{k}^{\prime}, q_{k}^{\prime}\right)$ corresponds to shape-stable conditions, we compare $u^{\prime}$ with $u$ : when $u^{\prime}>u$, the set $\left(p_{k}, q_{k}\right)$ is replaced by $\left(p_{k}^{\prime}, q_{k}^{\prime}\right)$ and the process is repeated. If $u^{\prime}$ is smaller than $u$, then the set $\left(p_{k}^{\prime}, q_{k}^{\prime}\right)$ is not automatically discarded as in other methods, but is accepted with a probability $\exp \left[\left(u^{\prime}-u\right) / \Theta\right]$, where $\Theta$ is a pseudotemperature that is gradually decreased as the iterations converge to the desired maximum. Typically $\Theta \simeq 10$ at the beginning of the process and is progressively decreased as described in Ref. [33]. The process is stopped when $\Theta$ has reached a value of the order of $10^{-2}$.

We have found that, depending on the rate of annealing, the estimated optimal point varies somewhat, as will be discussed further below. However, in all cases, we have been able to considerably increase the peak temperature over what is achievable with a single-frequency drive.

When the maximum $u$ has been found, we check that the higher-order shape modes $a_{n}$, with $n$ up to five, are also stable, again by calculating the pertinent Floquet multipliers. Typically, we find that the multipliers for $a_{3}$ and possibly $a_{4}$ are somewhat larger than those for $a_{2}$, although still stable, while those for $a_{5}$ and the higher modes are much smaller. At the optimum point $\left(p_{k}, q_{k}\right)$ we also calculate the concentration of dissolved gas with which the bubble would be in diffusional equilibrium $[6,7]$ and we check that the diffusional stability condition (17) is satisfied. In all cases, we have found that this condition was satisfied.

\section{RESULTS}

We demonstrate the results that are obtainable by the present method by considering a specific example with only three harmonic amplitudes $\left(p_{1}, p_{2}, q_{2}\right)$, so that

$$
P_{A}=p_{1} \cos \omega t+p_{2} \cos (2 \omega t)+q_{2} \sin (2 \omega t) .
$$

The fundamental frequency $\omega_{1} / 2 \pi$ is taken as $26.5 \mathrm{kHz}$ (which is of the order of the frequency used in much of the experimental work conducted to date $[2,4,34,35])$.

We have generated results using two different annealing protocols, with the second one proceeding three times as fast. Figure 1 compares the compression ratio $s=R_{\max } / R_{\min }$ and the maximum temperature $T_{\max }$ achievable with a single-
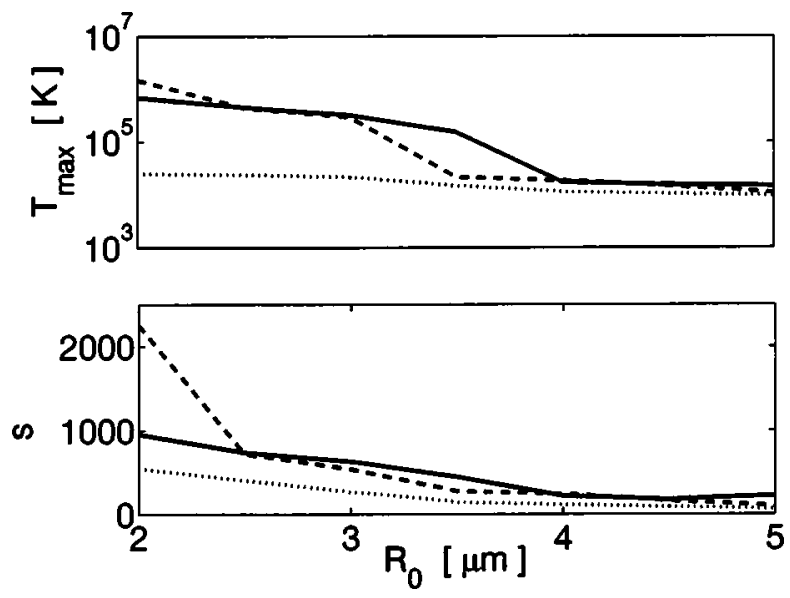

FIG. 1. Maximum collapse temperature and ratio $s$ $=R_{\max } / R_{\min }$ of the maximum bubble radius to the subsequent minimum as a function of the equilibrium radius for single-frequency drive (dotted line) and optimal multifrequency drive (dashed and solid lines, for the two different annealing rates mentioned in the text); the corresponding numerical values are given in Tables III and IV.

frequency drive (dotted lines) with the optima given by the two annealing procedures (solid and dashed lines), as a function of the bubble equilibrium radius $R_{0}$. The corresponding values of $\left(p_{1}, p_{2}, q_{2}\right)$ are given in Tables III and IV.

It is seen here that, for small bubbles $(\sim 2-3 \mu \mathrm{m})$, the optimum collapse temperature (upper graph) can be more than an order of magnitude larger than in the single-driving case. This is also reflected by the significantly increased compression ratio of these bubbles which is shown in the lower graph. The calculated temperatures for these very small bubbles, however, are very unlikely due to limitations of the model which become increasingly severe at such extreme conditions, notably the disregard of ionization and the idealized temperature and flux conditions conditions at the bubble wall.

These unrealistically large temperatures drastically drop with increasing $R_{0}$ although a clear enhancement over single-frequency drive is still present. For these larger bubbles, water vapor has a strongly adverse effect by increasing the heat capacity of the gas mixture and diverting an increasing part of the thermal energy to chemical reactions. In addition, larger bubbles tend to be more sensitive to shape distortions and, as a consequence, cannot be driven as strongly as small bubbles. Regardless of the equilibrium size, the time dependence of the radius is markedly different between one- and two-frequency driving; the upper panel of Fig. 2, in which the dashed and solid lines are for the singleand two-frequency drive, respectively, gives an example for $R_{0}=5 \mu \mathrm{m}$.

The bubble core temperature and argon mole fraction are also depicted in this figure. It can be seen here that, for the dual-frequency drive, the argon mole fraction at collapse is smaller than with a single frequency which, all other conditions being equal, would result in a smaller temperature. This circumstance, however, is more than compensated for by the greatly increased compression. 

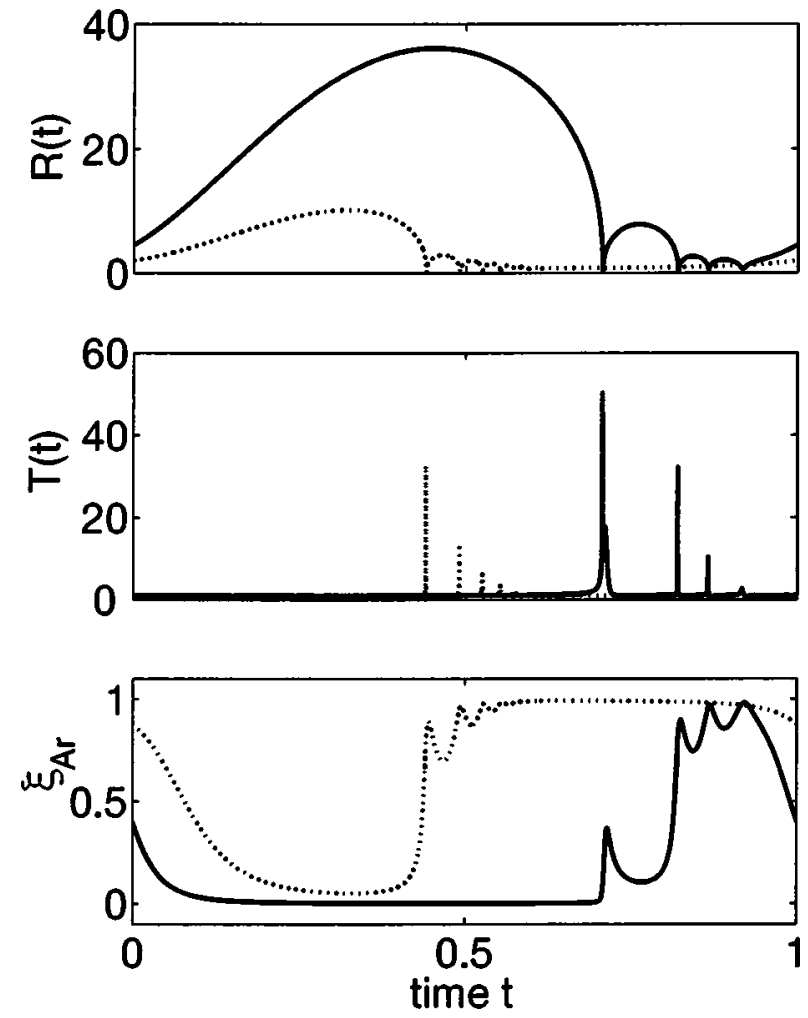

FIG. 2. Time dependence of the radius (normalized by its equilibrium value $R_{0}=5 \mu \mathrm{m}$ ) for single- (dashed line) and twofrequency drive in correspondence of the optimum conditions shown in Fig. 1 and Table III (upper panel), with the corresponding temperature [center panel, $T(t)$ normalized by $T_{0}=293.15 \mathrm{~K}$ ] and argon mole fraction histories.

The solid lines in Fig. 1 (which correspond to the results in Table III) are for the slow annealing rate, while the dashed lines are for the faster one. The differences between the two calculations are due to the exceedingly complex structure of the objective function, which would require annealing at a very slow rate for a resolution of these differences. We have not attempted this due to the large computational cost. In any event, the factor of 2 difference for $R_{0}=2 \mu \mathrm{m}$ cannot be regarded as significant due to very strong sensitivity of the results to small change in conditions such as pressure amplitudes, relative phase, and others. A further illustration of this sensitivity are the results for $R_{0}=3.5 \mu \mathrm{m}$, which are due to a particularly steep structure of the response surface in this neighborhood. As shown by a comparison of the results in Tables III and IV, for the larger values of the radius, the data are not significantly different in the two cases, and it is for these cases that the model is more realistic.

In practice, in order to observe the predicted effect, the bubble must be diffusively stable. The dissolved argon concentrations $C_{\infty}$ (normalized by the saturation concentration $C_{\text {sat }}$ ) necessary to maintain diffusional equilibrium at the optimum point for each bubble radius are tabulated in Tables III and IV, and graphed in Fig. 3; here the abscissa is the intensity of the sound field, $P_{\text {snd }}:=\sqrt{p_{1}^{2}+p_{2}^{2}+q_{2}^{2}}$. It will be noticed that the relative argon saturation necessary to observe the effects that we find are extremely low; they could

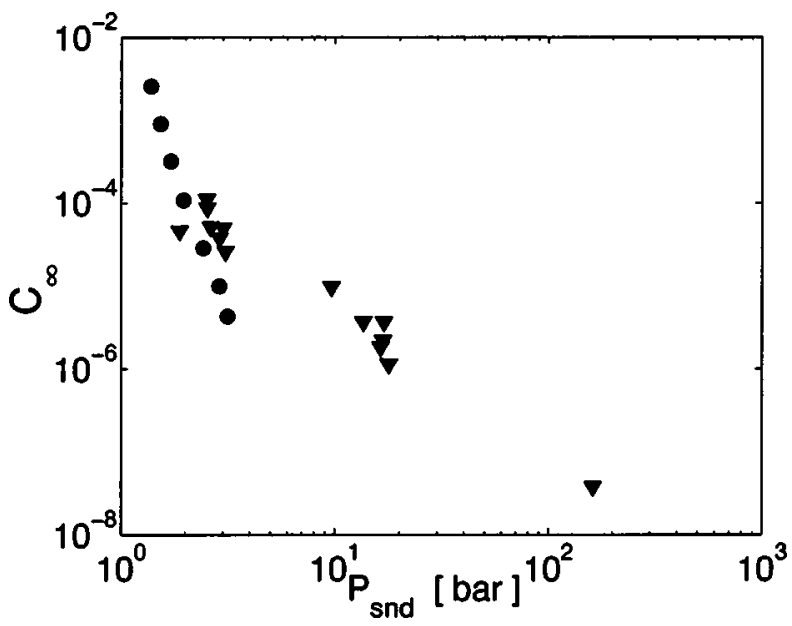

FIG. 3. Dissolved argon concentration (normalized by the saturation value $C_{\text {sat }}$ ) necessary to ensure diffusional equilibrium of the bubble as a function of the intensity of the sound field $P_{\text {snd }}$ $:=\sqrt{p_{1}^{2}+p_{2}^{2}+q_{2}^{2}}$. The circles are for single-frequency and the triangles for two-frequency drive. The diffusional equilibria are all found to be stable.

be achieved, for instance, by repeated dilution of the gas mixture to which the liquid is exposed.

All the single-frequency (circles) and multiple-frequency (triangles) data correspond to shape-stable conditions, and for all of them the diffusional equilibrium is also stable.

\section{REACHING THE OPTIMUM POINT}

While the results described prove the existence of a point in parameter space where the oscillation amplitude of a stable bubble is greatly increased over that attainable with a single-frequency drive, in order to exploit this finding in practice it is necessary to be able to reach this optimum point starting from a low drive amplitude while maintaining bubble stability. It is evident that this is a nontrivial requirement for the practical application of multifrequency enhancement of sonoluminescence. As a matter of fact, it is not even clear that such a path exists, as the maxima that we have identified may well belong to "islands" of stability that are not connected by stable paths to stable regions. Indeed, we have strong evidence of this possibility in at least one case, for the $2 \mu \mathrm{m}$ bubble in Table III. In this case, we observed that all steps away from the set of values shown in the table led to shape-unstable conditions. Furthermore, the Floquet multiplier for this case was very close to 1 . A similar complex topology of the stable-unstable regions is reflected in the results of [36] for the shape-stability boundary.

If, however, a stable path exists, a possible way to find it, which we have found useful, is the following, which we illustrate for a bubble with an equilibrium radius $R_{0}$ $=5 \mu \mathrm{m}$. Since the procedure is computationally intensive, for simplicity, we have carried out these computations excluding the chemical reactions from the model. This simplified model gives an optimum point different from that shown in Table III, with $p_{1}=157 \mathrm{kPa}, p_{2}=-121 \mathrm{kPa}, q_{2}$ $=54 \mathrm{kPa}$, and $C_{\infty, \text { opt }} / C_{\mathrm{sat}}=2 \times 10^{-4}$. In an experiment the 
dissolved gas concentration would be fixed, while the equilibrium radius of the bubble would vary as, for a fixed concentration, it depends on the acoustic drive.

We proceed backward starting from the optimum point and taking a small step $\delta \mathbf{P}=\left(\delta p_{1}, \delta p_{2}, \delta q_{2}\right)$ in the parameter space spanned by the three acoustic pressure amplitudes of Eq. (21) according to

$$
\delta \mathbf{P}=-\epsilon R_{0} \frac{\nabla R_{0}}{\left|\nabla R_{0}\right|^{2}},
$$

where the gradient is with respect to the pressure amplitudes and is taken keeping $C_{\infty}=C_{\infty, \text { opt }}$ constant; $\epsilon$ is a prescribed small number (typically of the order of $10^{-4}$ ). This procedure has the effect of changing the driving amplitudes in the direction $-\nabla R_{0}$, which is motivated by the general consideration that spherical stability improves for smaller bubbles. Furthermore, with single-frequency driving and at constant $C_{\infty}$, the value of $R_{0}$ for diffusive stability decreases with the pressure amplitude (see, e.g., Ref. [6], Fig. 7, Ref. [7], Fig. 14, or Ref. [3], Fig. 30). Although $\nabla R_{0}$ is calculated keeping $C_{\infty}$ constant, after the displacement (22), this condition will not be satisfied exactly due to the nonlinearity of the concentration-pressures relation. Furthermore, it is desirable to keep the shape-stability Floquet multiplier $\lambda$ away from the stability limits \pm 1 to avoid getting too close to an instability region. Thus, after taking the step (22), we adjust the pressure amplitudes further by employing the ansatz

$$
\delta \mathbf{P}=\alpha \boldsymbol{\nabla} C_{\infty}+\beta \nabla \lambda,
$$

and determining $\alpha$ and $\beta$ by imposing that

$$
C_{\infty}+\delta \mathbf{P} \cdot \nabla C_{\infty}=C_{\infty, \text { opt }},
$$

where $C_{\infty, \text { opt }}$ is the concentration corresponding to the optimum point, and

$$
\lambda+\delta \mathbf{P} \cdot \nabla \lambda=0 .
$$

The gradients in these two relations are taken with respect to pressure, keeping $R_{0}$ constant. The first condition is a Newton-Raphson extrapolation to the required value of $C_{\infty}$. If $\lambda$ were a linear function of $\delta \mathbf{P}$, the condition (25) would force it to vanish at the end of the step. In view of the nonlinearity of the $\lambda-\delta \mathbf{P}$ relation, (25) only forces the step to be in the direction of decreasing $|\lambda|$, i.e., more stable conditions. This adjustment is repeated until $|\lambda|<0.5$ and $C_{\infty}$ is within $1 \%$ of $C_{\infty, \text { opt }}$.

The same procedure is repeated until the pressure amplitudes are sufficiently small that the bubble is in a fully stable region.

The results of this procedure are shown in the threedimensional parameter space $\left(p_{1}, p_{2}, q_{2}\right)$ in Fig. 4.

At each point of this path the condition (17) of diffusive stability is satisfied. It can be seen in the figure that the path is complex and could not readily be found experimentally by trial and error. A good theoretical model appears therefore to

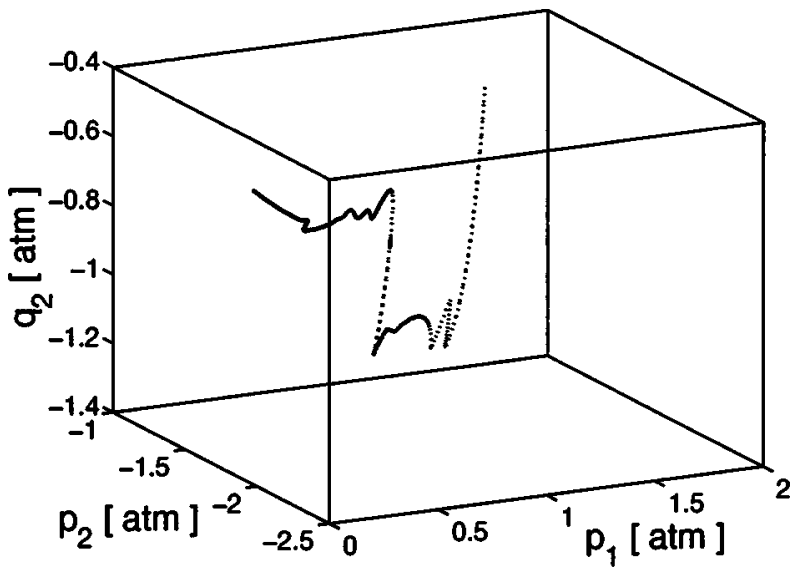

FIG. 4. Calculated diffusionally stable path from the stable region at low driving amplitudes to the optimum point for $R_{0}$ $=5 \mu \mathrm{m}$. The argon saturation is $C_{\infty} / C_{\text {sat }}=2 \times 10^{-4}$ and the fundamental driving frequency $f=26.5 \mathrm{kHz}$. The solid portion of the line denotes the region where the bubble is shape stable both with and without chemical reactions; on the dotted portion of the line spherical stability is predicted without chemical reactions only. Along the path the Floquet multiplier is constrained to be less than 0.5 in modulus.

be a prerequisite for any attempt at a full exploitation of the enhancement that multifrequency drive is predicted to render possible.

Once the path has been found, we can go back to the full model, including chemical reactions, and test again for spherical stability. The results of this test are shown in the same Fig. 4, where the solid part of the line marks the stable portion of the path, while the dotted part indicates spherically unstable conditions in the presence of chemical reactions. Somewhat unexpectedly, it is found that the latter destabilize the upper portion of the path. This finding is yet another demonstration of the subtle effect of the afterbounces on the shape stability of the bubble. Including chemical reactions, the last stable point of the path corresponds to $R_{0}$ $=2.6 \mu \mathrm{m}, \quad p_{1}=17.4 \mathrm{kPa}, \quad p_{2}=-209 \mathrm{kPa}, \quad q_{2}=-62 \mathrm{kPa}$, and a maximum temperature of $13800 \mathrm{~K}$.

It is possible that a stable path with chemical reactions could be found in the neighborhood of the one shown in Fig. 4. We have not pursued the matter in view of the considerable amount of computation required, which would have to be repeated in any attempt to investigate the issue experimentally. This paper is meant to point out the existence of optimum points at much higher pressure amplitudes than with single-drive excitation and to demonstrate computational approaches for their calculation.

\section{CONCLUSIONS}

It is known that single-bubble sonoluminescence can be enhanced by a proper selection of the liquid-gas combination, degree of liquid saturation, and operation at low temperatures [37-40]. We have demonstrated in this paper that there is a possibility of further significant enhancement by the use of an optimized set of Fourier amplitudes of the driving sound field, while maintaining shape, diffusive, and 
chemical stability. The effect is particularly marked for smaller bubbles, where we find gas temperatures much in excess of those achievable with single-frequency driving. While the upper range of the predicted temperatures for these small bubbles is unlikely due to limitations of the model (neglect of ionization, idealized bubble wall conditions, and others), for larger bubbles, where the model is more reliable, we find temperature increases by a factor of 2 or more. For these larger bubbles the increase is less marked as the influence of water vapor is more pronounced.

For the experimental observations of our predictions it will be necessary to gradually adjust the level of the Fourier components of the sound field so as to reach the optimum point while maintaining stability. We have shown a procedure for this purpose.

A final condition necessary to observe the predicted optimum conditions experimentally is that the pressure-radiation (or Bjerknes) force not lead to a removal of the bubble from the pressure antinode region. This point is a concern as it is well known that, as the maximum radius increases, the col- lapse of the bubble is delayed so much that the Bjerknes force may change sign and push a bubble driven below resonance away from the pressure antinode [41,42]. While this may happen, it is possible to avoid this difficulty, for example, by the simultaneous use of a very high frequency, which would greatly increase the pressure gradient responsible for the Bjerknes force, with little effect on the radial dynamics (see, e.g., Ref. [43]). It may also be noted that the condition of positional stability under the action of Bjerknes forces can readily be introduced as an additional constraint on the optimization algorithm.

\section{ACKNOWLEDGMENTS}

The work of X.L. and A.P. has been supported by DARPA. The work of R.T. and D.L. is part of the research program of the Stichting voor Fundamenteel Onderzoek der Materie (FOM), which is financially supported by the Nederlandse Organisatie voor Wetenschappelijk Onderzoek (NWO).
[1] D. Gaitan and L. Crum, in Frontiers in Nonlinear Acoustics, edited by M. Hamilton and D. Blackstock (Elsevier, New York, 1990), pp. 459-463.

[2] D. Gaitan, L. Crum, C. Church, and R. Roy, J. Acoust. Soc. Am. 91, 3166 (1992).

[3] M. Brenner, S. Hilgenfeldt, and D. Lohse, Rev. Mod. Phys. 74, 425 (2002).

[4] B. Barber, R. Hiller, R. Löfstedt, S. Putterman, and K. Weninger, Phys. Rep. 281, 65 (1997).

[5] M. Brenner, D. Lohse, and T. Dupont, Phys. Rev. Lett. 75, 954 (1995).

[6] S. Hilgenfeldt, D. Lohse, and M. Brenner, Phys. Fluids 8, 2808 (1996); for larger and better printed figures see ibid. 9, 2462(E) (1997)

[7] A. Prosperetti and Y. Hao, Philos. Trans. R. Soc. London, Ser. A 357, 203 (1999).

[8] H. Lin, B.D. Storey, and A.J. Szeri, Phys. Fluids 14, 2925 (2002).

[9] B.D. Storey and A.J. Szeri, Proc. R. Soc. London, Ser. A 456, 1685 (2000).

[10] B.D. Storey and A.J. Szeri, Proc. R. Soc. London, Ser. A 457, 1685 (2001).

[11] K. Yasui, J. Phys. Soc. Jpn. 66, 2911 (1997).

[12] R. Toegel, B. Gompf, R. Pecha, and D. Lohse, Phys. Rev. Lett. 85, 3165 (2000).

[13] J. Holzfuss, M. Rüggeberg, and R. Mettin, Phys. Rev. Lett. 81, 1961 (1998).

[14] J. Ketterling and R. Apfel, J. Acoust. Soc. Am. 107, 819 (2000).

[15] K. Hargreaves and T. Matula, J. Acoust. Soc. Am. 107, 1774 (2000).

[16] F. Moraga, R. Taleyarkhan, R.J. Lahey, and F. Bonetto, Phys. Rev. E 62, 2233 (2000).

[17] W. Chen, X. Chen, M. Lu, G. Miao, and R. Wei, J. Acoust. Soc. Am. 111, 2632 (2002).
[18] D. Krefting, R. Mettin, and W. Lauterborn, J. Acoust. Soc. Am. 112, 1918 (2002).

[19] A. Prosperetti and X. Lu, Riv. Ital. Acust. 25, 317 (2001).

[20] R. Toegel, S. Hilgenfeldt, and D. Lohse, Phys. Rev. Lett. 88, 034301 (2002).

[21] R. Toegel and D. Lohse, J. Chem. Phys. 118, 1863 (2003).

[22] B.D. Storey and A.J. Szeri, Phys. Rev. Lett. 88, 074301 (2002).

[23] J. Keller and M. Miksis, J. Acoust. Soc. Am. 68, 628 (1980).

[24] D.R. Lide, Handbook of Chemistry and Physics (CRC Press, Boca Raton, 1991).

[25] No literature values on the binary diffusion coefficient of argon and water vapor could be found, hence we calculate it from Ref. [44].

[26] E.U. Condon and H. Odishaw, Handbook of Physics (McGraw-Hill, New York, 1958).

[27] G.N. Hatsopoulos and J. Keenan, Principles of General Thermodynamics (Wiley, New York, 1965).

[28] M. Fyrillas and A. Szeri, J. Fluid Mech. 277, 381 (1994).

[29] M. Plesset and A. Prosperetti, Annu. Rev. Fluid Mech. 9, 145 (1977).

[30] V. Bogoyavlenskiy, Phys. Rev. E 62, 2158 (2000).

[31] A. Prosperetti, Atti Accad. Naz. Lincei, Cl. Sci. Fis., Mat. Nat., Rend. 62, 196 (1977).

[32] E. Coddington and N. Levinson, Theory of Ordinary Differential Equations (McGraw-Hill, New York, 1955).

[33] W. Goffe, G. Ferrier, and J. Rogers, J. Econometr. 60, 65 (1994).

[34] J. Ketterling and R. Apfel, Phys. Rev. Lett. 81, 4991 (1998).

[35] J. Ketterling and R. Apfel, Phys. Rev. E 61, 3832 (2000).

[36] Y. Hao and A. Prosperetti, Phys. Fluids 11, 1309 (1999).

[37] R. Hiller, K. Weninger, S. Putterman, and B. Barber, Science 265, 248 (1994).

[38] B. Barber, K. Wu, R. Löfstedt, P. Roberts, and S. Putternman, Phys. Rev. Lett. 72, 1380 (1994).

[39] S. Hilgenfeldt, D. Lohse, and W. Moss, Phys. Rev. Lett. 80, 
1332 (1998); 80, 3164(E) (1998).

[40] G. Simon, I. Csabai, A. Horváth, and F. Szalai, Phys. Rev. E 63, 026301 (2001).

[41] T. Matula, S. Cordry, R. Roy, and L. Crum, J. Acoust. Soc. Am. 102, 1522 (1997).

[42] I. Akhatov, R. Mettin, C. Ohl, U. Parlitz, and W. Lauterborn, Phys. Rev. E 55, 3747 (1997).

[43] K. Ohsaka and E. Trinh, J. Acoust. Soc. Am. 107, 1346 (2000).
[44] J.O. Hirschfelder, C.F. Curtiss, and R.B. Bird, Molecular Theory of Gases and Liquids (CRC Press, Boca Raton, 1991).

[45] G.P. Smith, G.D.M., M. Frenklach, N.W. Moriaty, B. Eiteneer, M. Goldenberg, C.T. Bowman, R.K. Hanson, S. Songand, W.C. Gardiner, Jr., V.V. Lissianski, and Z. Qin, GRI-Mech 3.0, http://www.me.berkeley.edu/gri_mech/

[46] J.A. Fay, Molecular Thermodynamics (Addison-Wesley, Reading, MA, 1965). 